Birgit Preuß-Scheuerle

Praxishandbuch Kommunikation 
Birgit Preuß-Scheuerle

\section{Praxishandbuch Kommunikation}

- Überzeugend auftreten

- Zielgerichtet argumentieren

- Souverän reagieren 
Bibliografische Information Der Deutschen Bibliothek

Die Deutsche Bibliothek verzeichnet diese Publikation in der Deutschen Nationalbibliografie; detaillierte bibliografische Daten sind im Internet über <http://dnb.ddb.de> abrufbar.

1. Auflage November 2004

Alle Rechte vorbehalten

(C) Betriebswirtschaftlicher Verlag Dr. Th. Gabler/GWV Fachverlage GmbH, Wiesbaden 2004

Lektorat: Diane Keller

Der Gabler Verlag ist ein Unternehmen von Springer Science+Business Media.

www.gabler.de

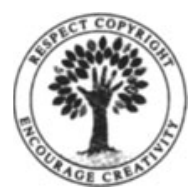

Das Werk einschließlich aller seiner Teile ist urheberrechtlich geschützt. Jede Verwertung außerhalb der engen Grenzen des Urheberrechtsgesetzes ist ohne Zustimmung des Verlags unzulässig und strafbar. Das gilt insbesondere für Vervielfältigungen, Übersetzungen, Mikroverfilmungen und die Einspeicherung und Verarbeitung in elektronischen Systemen.

Die Wiedergabe von Gebrauchsnamen, Handelsnamen, Warenbezeichnungen usw. in diesem Werk berechtigt auch ohne besondere Kennzeichnung nicht zu der Annahme, dass solche Namen im Sinne der Warenzeichen- und Markenschutz-Gesetzgebung als frei zu betrachten wären und daher von jedermann benutzt werden dürften.

Umschlaggestaltung: Digital Design Borgers $\mathrm{GmbH}$, Hünstetten

Satz: Fromm MediaDesign, Selters

Gedruckt auf säurefreiem und chlorfrei gebleichtem Papier

ISBN-13: 978-3-409-12676-2

e-ISBN-13: 978-3-322-84556-6

DOI: $10.1007 / 978-3-322-84556-6$ 


\section{Widmung}

Meinen Eltern 


\section{Danksagung}

Ich danke meinem Mann Olaf und meiner Mutter Elisabeth für die Alltagsarbeit, die sie mir abgenommen haben, meinem Vater Günter, der sich zeitweise allein versorgen musste, meinen Söhnen Till und Felix, die auf viel Spielzeit mit mir verzichtet haben und mich durch einfühlsame Fragen immer wieder neu zum Schreiben motivierten.

Dank geht auch an meine Freundin Tina für ihre konstruktive Kritik und meine KollegInnen Waltraud, Eva und Rainer für ihre fachliche Unterstützung.

Ganz besonders danke ich allen SeminarteilnehmerInnen, die in meinen Seminaren Fragen gestellt und Themen eingebracht haben und mir erlaubten, die Rollenspieldialoge zu verwenden. Ohne sie wäre das Buch nicht geschrieben worden.

Vielen Dank auch an das Lektorat von working@office, das mich von der Idee bis zum Druck immer gut begleitet hat. 


\section{Vorwort}

„Man kann nicht nicht kommunizieren.“

Paul Watzlawik

Seit 15 Jahren, in denen ich mit SekretärInnen, TeamassistentInnen, SachbearbeiterInnen und Führungskräften trainiere, begegnen mir zum Thema Kommunikation immer wieder dieselben Fragen:

Wie wirke ich? Wie überzeuge ich? Wie kommuniziere ich so, dass andere verstehen, was ich sage? Wie soll ich mich verhalten, wenn andere sich unfair verhalten? Wie gehe ich mit Konflikten um?

Natürlich haben die Fragestellungen, je nach beruflicher Situation der Teilnehmenden, einen anderen Fokus, im Kern sind sie jedoch alle gleich. Die Grundsatzfrage ist: Wie funktioniert Kommunikation?

Deshalb können Sie in diesem Buch zuerst Informationen über die Körpersprache und die Stimme, über das Zuhören, die Psychologie der Kommunikation, das Argumentieren und die Gesprächsführung lesen. Die verschiedenen Kommunikationsmodelle tragen in unterschiedlichen Situationen zur Verbesserung der Kommunikation bei.

Im zweiten Teil des Buches werden dann verschiedene Kommunikationssituationen vorgestellt. Wie kommunizieren Sie mit Ihren Vorgesetzten, mit KollegInnen, KundInnen und Dienstleistern, und wie am Telefon? Die Beispiele sind aus dem beruflichen Alltag der SeminarteilnehmerInnen und wurden in den Seminaren diskutiert und analysiert.

Viele Übungen und Checklisten geben Ihnen die Möglichkeit, Ihren Kommunikationsstil $\mathrm{zu}$ analysieren und klarer, zielgerichteter und überzeugender zu kommunizieren.

Sie können das Buch von der ersten bis zur letzten Seite lesen oder einfach nach Lust, Laune und Fragestellung in einzelne Kapitel eintauchen. Durch die Verweise zu anderen Kapiteln erhalten Sie ein umfassendes Bild über die tägliche Kommunikation an Ihrem Arbeitsplatz.

Die Umsetzungstipps im letzten Kapitel unterstützen Sie auf dem Weg von der Theorie zur Praxis.

Ich wünsche Ihnen viel Spaß beim Lesen und viel Erfolg beim Üben und Umsetzen!

\section{Birgit Preuß-Scheuerle}

www.bps-training.de 


\section{Inhaltsverzeichnis}

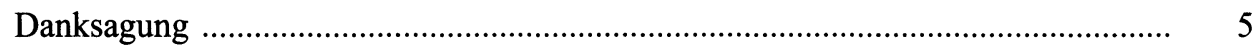

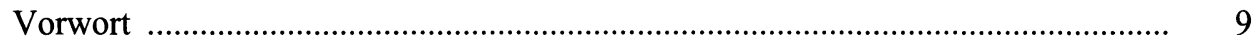

1 Die verschiedenen Ebenen der Kommunikation .......................................... 15

1.1 Die Inhaltsebene und die Beziehungsebene ………............................... 15

1.2 Wirkungsfaktoren in der Kommunikation ................................................ 16

1.2.1 Übung - Interpretation der Körpersprache …………………........ 18

1.2.2 Die Körpersprache als Interpretationshilfe................................... 24

1.2.2.1 Abstand schafft Verständnis ......................................... 24

1.2.2.2 Haltung gibt Halt ........................................................ 26

1.2.2.3 Gestik betont ............................................................... 27

1.2.2.4 Mimik zeigt Gefühl .................................................... 28

1.2.2.5 Übung - Wahrnehmung der Körpersprache .................. 30

1.2.3 Die Stimme als Interpretationshilfe ............................................... $\quad 30$

1.2.3.1 Stimmübungen ............................................................ 32

1.2.4 Erfolgskontrolle im Gespräch .................................................... 33

1.2.5 Übung - Interpretation eines Dialoges ……………………….... 34

2 Verschiedene Arten des Zuhörens ……………........................................ 37

2.1 Das Ich-verstehe-Zuhören .................................................................. 37

2.2 Das aufnehmende Zuhören ................................................................... 38

2.3 Das umschreibende Zuhören ................................................................. 39

2.3.1 Übung - kontrollierter Dialog …………………….................... 42

2.4 Das aktive Zuhören ............................................................................ 43

2.4.1 Übung - aktives Zuhören .......................................................... 44

3 Psychologie der Kommunikation .......................................................... 47

3.1 Alles hat vier Seiten - das Nachrichten-Quadrat ................................... 47

3.1.1 Übung - alle-vier-Seiten-aussprechen .......................................... 49

$3.2 \quad$ Alle haben vier Ohren ....................................................................... $\quad 50$

3.2.1 Übung - auf-welchem-Ohr-hören-Sie? ..................................... 51

3.3 Umgang mit den eigenen Emotionen ....................................................... 53

3.3.1 Die Transaktionsanalyse (TA) .................................................. 54

3.3.2 Das Internal-Family-System (IFS) ……………………………... 59

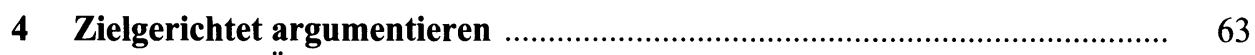

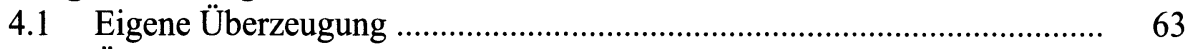

4.2 Überzeugende Körpersprache und Stimme ……....................................... 63

4.3 Klare Argumentation ......................................................................... 64

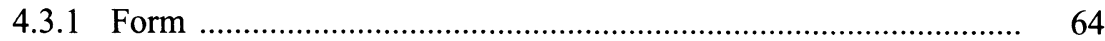

4.3.2 Inhalt ....................................................................... 64

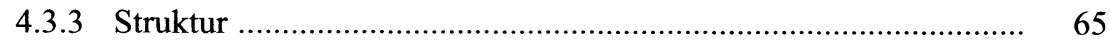


4.4 Direkte Ansprache und Motivation ...................................................... 71

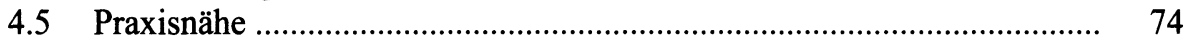

4.6 Klare Bitten, Aufforderungen und Appelle ....................................... 75

4.7 Checkliste Argumentation .............................................................. 76

5 Gesprächsführung ................................................................................. 77

5.1 Gemeinsamkeiten aller Gespräche ................................................ 77

5.2 Gesprächsvorbereitung ............................................................... 78

5.3 Checkliste Gesprächsvorbereitung …................................................ 80

5.4 Wie verläuft ein Gespräch? .............................................................. 80

5.5 Wer fragt, führt - aber wie? ........................................................ 83

5.5.1 Fragen sinn- und wirkungsvoll eingesetzt ................................ 83

5.5.2 Wie stellen Sie Fragen? ....................................................... 85

5.6 Positiv formulieren ........................................................................ 87

5.6.1 Von unklaren zu klaren Formulierungen .................................. 88

5.6.2 Von negativen zu positiven Formulierungen ............................. 89

5.6.3 Von passiven zu aktiven Formulierungen ................................ 91

5.6.4 Von vergangenheits- zu zukunftsorientierten Formulierungen ..... 92

5.6.5 Von verurteilenden zu wertschätzenden Formulierungen ............ 92

5.7 Nutzen Sie Gesprächsförderer ...................................................... 94

5.8 Meiden Sie Gesprächsfallen .............................................................. 94

5.9 Erkennen Sie Fallstricke ............................................................ 95

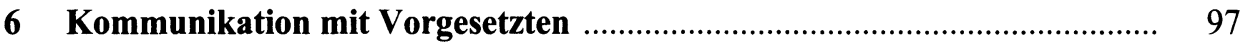

6.1 Meine Chefin/mein Chef ist ............................................................. 98

6.2 Meine Chefin/mein Chef gibt kein positives Feedback ......................... 102

6.3 Wie sage ich es meiner Chefin/meinem Chef? .................................... 106

6.3.1 Trainieren Sie das Nein-Sagen .............................................. 109

6.4 Wer sagt es den Vorgesetzten? ........................................................ 109

6.4.1 Checkliste „Wie sage ich es meiner Chefin/meinem Chef?““ ....... 110

6.4.2 Übung - die Umstrukturierung .............................................. 110

6.5 Einmal im Jahr - das MitarbeiterInnengespräch .............................. 115

6.5.1 Übung - Frau Reimers „Orientierungsgespräch“ ...................... 116

7 Kommunikation mit den KollegInnen ................................................. 119

7.1 Im Auftrag des Vorgesetzten delegieren ............................................ 119

7.2 Frauen und Männer - ein kleiner Unterschied ................................. 122

7.3 Konfliktanalysen ................................................................. 122

7.3.1 Woran erkennen Sie einen Konflikt? .................................... 123

7.3.2 Konfliktarten und Ursachen ................................................. 123

7.3.3 Konfliktebenen ................................................................. 125

7.3.4 Netzwerkdiagramm ......................................................... 125

7.3.5 Eskalationsstufen eines Konflikts ........................................... 127

7.3.6 Konfliktlösungsstile ............................................................. 130

7.3.7 Handlungsmöglichkeiten bei der Konfliktlösung ....................... 134

7.3.8 Fünf Fragen für die Ergebnisbewertung von Konfliktlösungen .... 134

7.3.9 Konfliktlösungsrahmen ....................................................... 135 
7.4 Das Klärungsgespräch .................................................................... 136

7.4.1 Wie führen Sie ein Klärungsgespräch? ...................................... 137

7.4.2 Kritik äußern, ohne zu verletzen - gewaltfrei ............................ 138

7.4.3 Zwölf Fallen bei Klärungsgesprächen ......................................... 142

7.4.4 So reagieren Sie, wenn mit Ihnen ein Klärungsgespräch geführt wird

7.5 Übung - Beruf ist nicht gleich Beruf - alte Standesdünkel ......................... 144

7.6 Übung - die neue Teamkollegin - Harmonie um jeden Preis? ................. 145

8 Kommunikation mit KundInnen ............................................................. 149

8.1 Ihre persönliche KundInnen-Wunsch-Liste ........................................... 153

8.2 Gelassenheit ist eine Frage des Denkens ............................................ 156

8.3 Und wenn Sie sich trotzdem ärgern? .................................................. 157

8.4 Sagen Sie auch zu KundInnen höflich Nein .......................................... 159

8.5 Übung-Reklamationsgespräch ............................................................ 161

9 Kommunikation mit DienstleisterInnen ................................................... 165

9.1 Ihre Verhandlungskompetenzen ....................................................... 166

9.2 Ihr persönlicher Verhandlungsstil ........................................................... 167

9.2.1 Übung - Ihr persönlicher Verhandlungsstil .................................. 169

9.3 Verhandlungstypen .......................................................................... 170

9.4 Checkliste zur Vorbereitung auf Verhandlungen .................................. 172

9.5 Gespräche ohne VerliererInnen ........................................................ 173

9.6 Übung - Verhandlung mit einer Referentin ....................................... 178

9.7 Übung - Verhandlung mit einem Hotel ................................................ 181

10 Kommunikation am Telefon .............................................................. 185

10.1 Besonderheiten am Telefon ............................................................ 185

10.1.1 Nachteile des Telefons ............................................................ 186

10.1.2 Vorteile des Telefons ……………………............................. 187

10.2 Professionell telefonieren ................................................................ 187

10.2.1 Checkliste für Telefongespräche ……....................................... 189

10.2.2 Stimmen Sie sich ein ............................................................ 191

10.2.3 Der Einstieg ist entscheidend ................................................. 191

10.2.4 Während des Gesprächs ............................................................. 192

10.2.5 Zielstrebig das Gespräch beenden ............................................ 193

10.2.6 Umgang mit Reklamationen ..................................................... 193

10.2.7 Steigen Sie bei Beschimpfungen aus ......................................... 194

10.3 Übung - Telefonat mit einem Anbieter ……………………………..... 195

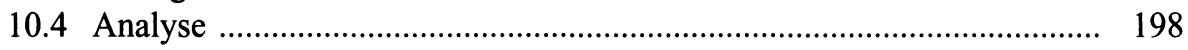

11 Umsetzung …….............................................................................. 201

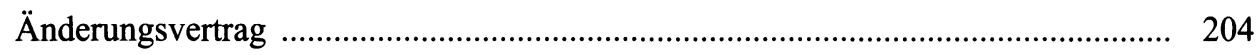

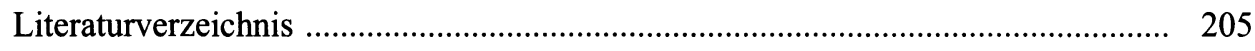

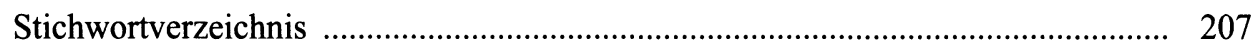

